\title{
EWSR1 Rearrangement and CD99 Expression as Diagnostic Biomarkers for Ewing/PNET Sarcomas in a Moroccan Population
}

\author{
Sara Louati $\mathbb{D}^{1,2}{ }^{1,2}$ Nadia Senhaji, ${ }^{1}$ Laila Chbani, ${ }^{2,3}$ and Sanae Bennis ${ }^{2,3}$ \\ ${ }^{1}$ Bioactive Molecules, Structure and Functions Laboratory, Faculty of Sciences and Techniques, \\ Sidi Mohamed ben Abdellah University of Fez, Morocco \\ ${ }^{2}$ Pathological Anatomy and Molecular Pathology Department, Hassan II University Hospital of Fez, Morocco \\ ${ }^{3}$ Biomedical and Translational Research Laboratory, Faculty of Medicine and Pharmacy, \\ Sidi Mohamed ben Abdellah University of Fez, Morocco \\ Correspondence should be addressed to Sara Louati; sara.louati@yahoo.fr
}

Received 15 May 2018; Revised 16 August 2018; Accepted 18 August 2018; Published 18 September 2018

Academic Editor: Monica Cantile

Copyright (C) 2018 Sara Louati et al. This is an open access article distributed under the Creative Commons Attribution License, which permits unrestricted use, distribution, and reproduction in any medium, provided the original work is properly cited.

\begin{abstract}
Ewing sarcoma/primitive neuroectodermal tumor (Ewing/PNET sarcomas or EPS) are a group of round cell tumors. Malignant round cell tumors form a large and diverse group that includes rhabdomyosarcoma, synovial sarcoma, non-Hodgkin's lymphoma, neuroblastoma, hepatoblastoma, Wilm's tumor, desmoplastic small round cell tumor, and other morphologically similar entities. Differential diagnosis of Ewing sarcoma/primitive neuroectodermal tumor (Ewing/PNET sarcomas or EPS) is difficult. In addition to morphology and immunohistochemistry (IHC), differential diagnosis of these tumors is based on molecular analysis of the EWSR1 gene rearrangement using fluorescent in situ hybridization (FISH) technique. We investigated the diagnostic value of combined CD99 immunostaining and EWSR1 t(22q12) alteration using a dual-color, break-apart rearrangement probe in forty-one formalin-fixed paraffin-embedded (FFPE) tissue samples from pediatric and adult patients diagnosed with EPS. IHC was performed in all cases using the CD99 antibody and showed a positivity of $92.7 \%$ in the enrolled cases $(38 / 41)$ followed by FISH analysis where $48.8 \%$ of the cases $(20 / 41)$ were rearranged. Sensitivity and specificity for IHC assays were $88 \%$ and $58 \%$, respectively. Notably, FISH had a sensitivity of $100 \%$ and a specificity of $87 \%$. In addition, CD 99 positivity was found to correlate with EWSR1 rearrangement $(p<0.05)$. This report shows that FISH has better sensitivity and specificity than IHC in the Moroccan population, and supports its combination with CD99 immunostaining as diagnostic biomarkers for this rare malignant entity."
\end{abstract}

\section{Background}

Small round cell tumors are highly aggressive malignant tumors that are characterized by small and relatively monotonous, undifferentiated cells. This group consists of Ewing sarcoma, PNETs, rhabdomyosarcoma, synovial sarcoma, non-Hodgkin's lymphoma, retinoblastoma, neuroblastoma, hepatoblastoma, nephroblastoma, small cell osteogenic sarcoma, Wilm's tumor, and desmoplastic small round cell tumor. The undifferentiated or poorly differentiated primitive character of the tumor cells, as well as the rare occurrence of these tumors relative to the more differentiated and common carcinomas, makes differential diagnosis of small round cell tumor types particularly difficult. Ewing sarcoma (OMIM: 612219) was first described in 1921 by the American pathologist James Stephen Ewing (named as diffuse endothelioma of bone) [1]. This rare and aggressive tumor is characterized by small round cells that occur frequently in soft tissue and bone of adolescents and young adults [2]. Subsequently, it was noted that some of these tumors show features of neural differentiation (pseudorosettes of Homer-Wright and positive Periodic acid-Schiff (PAS) stain), which suggested the presence of a new histological subentity $[2,3]$. Tumor variants with these neural features have been named Ewing/PNET sarcomas (EPS). The identification of this entity in routine histopathological examinations has improved significantly with the emergence of IHC and molecular biology techniques. Most of EPS 
tumors express CD99, which is a highly sensitive immunohistochemical biomarker [4]. However, this marker lacks specificity and can be positive in other sarcomas and lymphomas [4]. In addition, CD57, synaptophysin, chromogranin, vimentin, neuron-specific enolase (NSE), and S-100 are often expressed in EPS tumors [2,4]. Importantly, these tumors show frequent and specific rearrangement of the Ewing sarcoma breakpoint region 1 (EWSR1) gene located on chromosome $22[5,6]$. Translocations between EWSR1 and Friend leukemia integration 1 transcription factor (FLI1) (85\%, t $11 ; 22)(\mathrm{q} 24 ; \mathrm{q} 12))$ and between EWSR1 and ETS transcription factor $(E R G)(10 \%, \mathrm{t}(21 ; 22)(\mathrm{q} 22 ; \mathrm{q} 12))$ are key differential cytogenetic alterations in EPS [7]. These chimeric fusions encode for multifunctional proteins encompassing various cancer hallmarks such as evading growth suppressors, sustaining proliferative signaling, resisting cell death, inducing angiogenesis, and activating invasion and metastasis which are believed to drive the tumorigenesis of this rare entity [5]. Specific FISH with break-apart probe targeting translocations on FFPE sections is commonly used in combination with CD99 immunostaining for diagnosis of these tumors.

Morocco harbors a unique population that is a complex admixture of autochthonous Maghrebi (including Berber-speaking groups) and European, Northwest African, West African, and West Asian lineages, with various degrees of intermixing. Here, we report the results of a study evaluating the sensitivity and specificity of FISH-based EWSR1 rearrangement, as well its correlation with CD99 positivity, for enhanced diagnosis of EPS in a large Moroccan cohort.

\section{Materials and Methods}

2.1. Patient Selection and Data Collection. The study protocol was approved by the ethics committee of Hassan II University Hospital of Fez, Morocco. FFPE tissue from cases coded as EPS was retrieved from the Pathology Department of Hassan II University Hospital of Fez, Morocco. All cases were histologically reviewed and the diagnosis of EPS was based on histology and IHC according to the latest World Health Organization (WHO) classification of tumors of soft tissue and bone [8]. Cases with insufficient or poor quality of tissue were excluded. A total of forty-one cases of pediatric and adult patients diagnosed with EPS were included in this study. In addition, cases with round cell liposarcomas $(n=5)$, desmoplastic small round cell tumors $(n=2)$, and embryonic rhabdomyosarcomas $(n=8)$ were included as comparative controls.

2.2. Immunohistochemical Analysis. IHC analysis was performed in all cases (41 cases of EPS and 15 controls). Sections ( $5 \mu \mathrm{m}$ thick) were prepared from FFPE tissue blocks and stained with hematoxylin, eosin, and safranin (HES). Immunostaining was performed with CD99 Rabbit Monoclonal Antibody (EPR3097Y, dilution: 1:100, Cell Marque ${ }^{\mathrm{TM}}$, Sigma-Aldrich ${ }^{\circledR}$ ) using the LSAB kit (DakoPatts ${ }^{\circledR}$, Copenhagen, Denmark) with an automated immunostainer. Pretreatment using a heat-induced epitope retrieval (HIER) procedure prior to IHC was performed and DAB (3,3'-diaminobenzidine tetrahydrochloride) was used as a chromogen. Appropriate positive and negative control samples were used thoroughly. Results were considered positive when $>10 \%$ membranous staining was observed in tumor cells.

2.3. Fluorescence In Situ Hybridization. The FISH analysis was performed in all cases (41 EPS cases and 15 controls). Sections $(3.5 \mu \mathrm{m}$ thick) from FFPE tissue were processed for FISH using the EWSR1 (22q12) dual-color break-apart rearrangement probe (Vysis FISH, Abbott Laboratories ${ }^{\circledR}$ ). The FISH was then performed according to the manufacturer's recommendation. In normal cells lacking $\mathrm{t}(22 \mathrm{q} 12)$, a two-fused signal pattern (yellow) is expected to reflect two intact copies of the EWSR1 gene. In abnormal cells with $\mathrm{t}(22 \mathrm{q} 12)$, a split signal is observed (one green and one orange signal pattern). For each sample, a minimum of 100 nonoverlapping tumor cells were evaluated to assess the presence of fused or split green and red signals. A positive result was defined as $>30 \%$ of cells with split signals.

2.4. Statistical Analysis. Sensitivities, specificities, positive predictive values (PPV), and negative predictive values (NPV) were calculated using the final pathologic diagnosis as the gold standard and defined as follows: sensitivity $=$ true positives/(true positives + false negatives); specificity $=$ true negatives/(true negatives + false positives); PPV = true positives/(true positives + false positives); NPV = true negatives/ (true negatives + false negatives). Epi Info ${ }^{\mathrm{TM}}$ software, version 7.2 , was used to perform all analyses. Youden's J statistic (Youden's Index $=($ sensitivity + specificity $)-1)$ [9] was used for diagnostic accuracy. A $\chi^{2}$ test was used to correlate CD99 positivity with the presence of EWSR1 rearrangement. The results are considered significant when $p<0.05$.

\section{Results}

3.1. Clinical Features. This retrospective study was conducted over 85 months (from January 2010 to January 2017). The average age of patients was 24 years, ranging from 3 to 62 years (median, 19 years) with male predominance (27 males/14 females). $29.3 \%$ of the cases (12/41) occurred in the lower limbs, $17.1 \%(7 / 41)$ in the thorax, $12.2 \%(5 / 41)$ in the abdomen, $12.2 \%$ (5/41) in the upper limbs, $12.2 \%(5 / 41)$ in the pelvis, and $2.4 \%(1 / 41)$ in the head and neck. For $14.6 \%(6 / 41)$ of the cases, the only information provided concerning the location was just the soft tissue. All EPS cases have been reviewed by a pathologist, diagnosed and graded III according to the FNCLCC (Fédération Nationale des Centres de Lutte Contre le Cancer) classification (Table 1).

3.2. Histopathology and IHC Analyses. EPS are composed of proliferating small round cells (Figure 1(a)). Mitotic activity ranged from 4 to 20 mitoses per 10 high power fields (median, 10 mitoses). Areas of tumor necrosis were present in $92.7 \%$ of the cases $(38 / 41)$, including 24 cases displaying more than $50 \%$ and 14 cases with less than $50 \% .92 .7 \%$ of the cases (38/41) showed a positivity for CD99 antibody, including 29 cases with strong, diffuse, and characteristic 
TABLE 1: Clinical features of enrolled 41 EPS cases.

\begin{tabular}{|c|c|c|c|c|}
\hline $\begin{array}{l}\text { Case } \\
\text { number }\end{array}$ & $\begin{array}{l}\text { Age and } \\
\text { sex }\end{array}$ & $\begin{array}{l}\text { Anatomic } \\
\text { site }\end{array}$ & $\begin{array}{l}\text { EWSR1 gene } \\
\text { rearrangement }\end{array}$ & $\begin{array}{c}\text { CD99 } \\
\text { staining }\end{array}$ \\
\hline 1 & $26 / \mathrm{M}$ & Abdomen & - & +++ \\
\hline 2 & $37 / \mathrm{M}$ & Lower limbs & - & + \\
\hline 3 & $10 / \mathrm{F}$ & Head and neck & + & +++ \\
\hline 4 & $40 / \mathrm{F}$ & Lower limbs & + & + \\
\hline 5 & $14 / \mathrm{M}$ & Soft tissue & - & +++ \\
\hline 6 & $16 / \mathrm{M}$ & Upper limbs & + & - \\
\hline 7 & $19 / \mathrm{M}$ & Lower limbs & + & + \\
\hline 8 & $10 / \mathrm{M}$ & Lower limbs & - & + \\
\hline 9 & $8 / \mathrm{F}$ & Lower limbs & + & +++ \\
\hline 10 & $19 / \mathrm{M}$ & Lower limbs & + & +++ \\
\hline 11 & $10 / \mathrm{M}$ & Pelvis & - & + \\
\hline 12 & $13 / \mathrm{M}$ & Upper limbs & + & +++ \\
\hline 13 & $38 / \mathrm{M}$ & Pelvis & - & +++ \\
\hline 14 & $6 / \mathrm{F}$ & Pelvis & IN & +++ \\
\hline 15 & $19 / \mathrm{F}$ & Upper limbs & + & +++ \\
\hline 16 & $8 / \mathrm{M}$ & Lower limbs & + & +++ \\
\hline 17 & $18 / \mathrm{M}$ & Abdomen & - & +++ \\
\hline 18 & $5 / \mathrm{M}$ & Thorax & + & +++ \\
\hline 19 & $9 / \mathrm{F}$ & Lower limbs & + & +++ \\
\hline 20 & $15 / \mathrm{M}$ & Lower limbs & - & +++ \\
\hline 21 & $22 / \mathrm{M}$ & Thorax & - & +++ \\
\hline 22 & $11 / \mathrm{M}$ & Soft tissue & - & +++ \\
\hline 23 & $23 / \mathrm{F}$ & Thorax & + & +++ \\
\hline 24 & $17 / \mathrm{M}$ & Thorax & + & +++ \\
\hline 25 & $6 / \mathrm{F}$ & Thorax & - & +++ \\
\hline 26 & $21 / \mathrm{M}$ & Soft tissue & - & +++ \\
\hline 27 & $18 / \mathrm{M}$ & Lower limbs & IN & + \\
\hline 28 & $21 / \mathrm{F}$ & Pelvis & + & +++ \\
\hline 29 & $62 / \mathrm{F}$ & Abdomen & - & + \\
\hline 30 & $28 / \mathrm{F}$ & Soft tissue & - & +++ \\
\hline 31 & $29 / \mathrm{M}$ & Abdomen & - & - \\
\hline 32 & $14 / \mathrm{M}$ & Lower limbs & + & +++ \\
\hline 33 & $25 / \mathrm{M}$ & Upper limbs & + & +++ \\
\hline 34 & $3 / \mathrm{M}$ & Lower limbs & + & +++ \\
\hline 35 & $29 / \mathrm{M}$ & Pelvis & + & +++ \\
\hline 36 & $28 / \mathrm{M}$ & Soft tissue & - & +++ \\
\hline 37 & $50 / \mathrm{F}$ & Soft tissue & + & +++ \\
\hline 38 & $40 / \mathrm{M}$ & Thorax & - & + \\
\hline 39 & $62 / \mathrm{F}$ & Upper limbs & - & + \\
\hline 40 & $37 / \mathrm{M}$ & Thorax & - & - \\
\hline 41 & $21 / F$ & Abdomen & + & +++ \\
\hline
\end{tabular}

For EWSR1 rearrangement: positive (+), negative (-), and inconclusive (IN); for CD9 immunostaining: positive staining $<50 \%(+)$ and strong, diffuse, and membranous staining $\geq 50 \%(+++)$.

membranous staining (Figure 1(b)). The CD99 antibody was negative in all 15 comparative controls.

3.3. Cytogenetic Analysis. The FISH analysis showed a rearrangement of the EWSR1 gene locus in $48.8 \%$ of the cases
(20/41) (Figure 1(c)). In 7.3\% of the cases (3/41), interpretation of hybridization signals was not possible because of weak signals, insufficient tissue, or poor tissue fixation. FISH was negative for all 15 cases used as comparative controls (Figure 1(d)).

3.4. Sensitivity, Specificity, NPV, and PPV. Table 2 summarizes sensitivities, specificities, PPV, NPV, and Youden's index for each of CD99 antibody and FISH with the EWSR1 (22q12) dual-color, break-apart rearrangement probe for the diagnosis of EPS. IHC of CD99 showed a positivity of $92.7 \%$ in the enrolled cases $(38 / 41)$. FISH showed that $48.8 \%$ of the cases $(20 / 41)$ were rearranged. Sensitivity and specificity for IHC assays were $88 \%$ and $58 \%$, respectively. Notably, FISH had a better sensitivity (100\%) and specificity (87\%). In terms of PPV, the FISH technique (89\%) predicts the disease better than IHC (48\%). The Youden's index of EWSR1 break-apart (0.9) was near the value of 1 which supports the FISH technique as an accurate diagnostic tool for EPS. Furthermore, we found a strong correlation between the diffuse positivity $\geq 50 \%$ of CD 99 and the presence of EWSR1 rearrangement $(p<0.05)$ (Table 3).

\section{Discussion}

Ewing sarcoma and PNET are aggressive round cell tumors and considered as two morphologic variants of the same entity (EPS) because they show similar clinical, immunohistochemical, and molecular profiles [7]. These tumors usually arise in bone and occasionally in deep soft tissues of the paraspinal region, the chest wall, and the lower extremities in children and young adults [8]. Currently, the EPS family is defined by the presence of specific translocations frequently involving the EWSR1 gene, which is fused to an E26 transformation-specific (ETS) family gene (FLI-1, ERG, or ETS variant 1 (ETV1)) [10]. In addition, they also express the membranous CD99 which is characteristic and sensitive but not specific [11]. A combination of histopathology examination, IHC, and FISH techniques is the gold standard for the diagnosis of EPS.

We have performed a comprehensive investigation of combined CD99 immunostaining and EWSR1 rearrangement as diagnostic biomarkers to increase their sensitivities and specificities in a large cohort of Moroccan EPS patients. In this study, we showed that combining IHC and FISH (CD99 and EWSR1 rearrangement) is more sensitive and specific than each test alone for the diagnosis of EPS. The dual-color, break-apart FISH technique is used in several pathology laboratories to identify the rearrangement of the EWSR1 gene with a potential added value in the diagnosis of EPS $[12,13]$. Furthermore, it is considered as a standard of choice because it can be easily performed in FFPE tissues with a good preservation of tissue architecture [14]. Previously, Mhawech-Fauceglia et al. found a sensitivity of 50\% in a similar study, which is significantly less than our result (100\%) [14]. This difference between the two studies could be explained by the number of recruited cases that can affect the statistical analysis and the conservation of the FFPE tissue or even the duration of sample fixation. The fixation is 


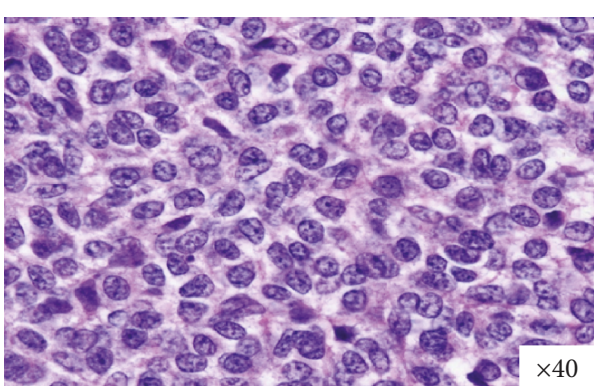

(a)

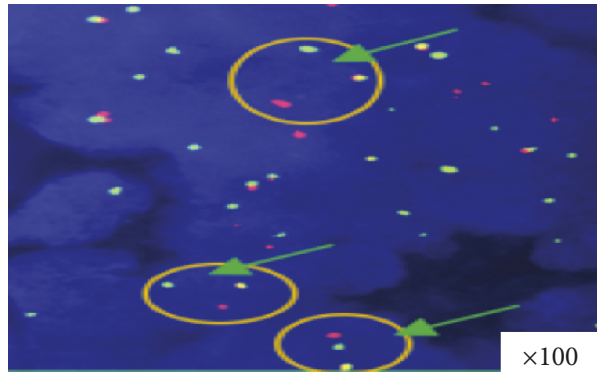

(c)

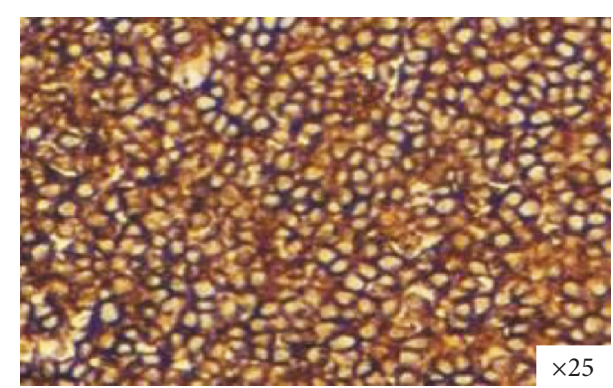

(b)

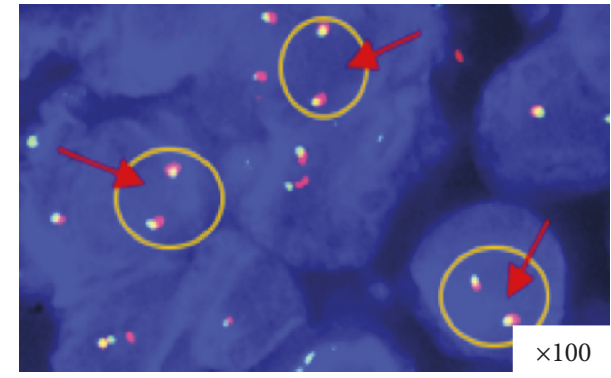

(d)

FIGURE 1: H\&E staining, CD99 immunostaining, and dual-color FISH for the EWSR1 gene in selected EPS cases. (a) H\&E stained section showing diffuse proliferation of round blue cells. (b) Diffuse, intense, and membrane expression of CD99 antibody. (c) Dual-color FISH performed with break-apart EWSR1 probes reveals nuclei in which one pair of the probe signals is split apart due to a rearrangement in the EWSR1 gene (green arrows). Red arrows indicate a two-signal pattern in normal cells (d).

TABLE 2: Comparison between IHC and FISH test for the diagnosis of EPS.

\begin{tabular}{lcc}
\hline & CD99 antibody & EWSR1 break apart \\
\hline Number of cases & 56 & 56 \\
Sensitivity & $88 \%$ & $100 \%$ \\
Specificity & $58 \%$ & $87 \%$ \\
PPV & $48 \%$ & $89 \%$ \\
NPV & $92 \%$ & $100 \%$ \\
Youden's index & 0,55 & 0,9 \\
\hline
\end{tabular}

Abbreviations: NPV: negative predictive value, PPV: positive predictive value.

TABLE 3: Association of EWSR1 rearrangement and CD99 positivity.

\begin{tabular}{|c|c|c|c|c|c|c|c|}
\hline \multirow{3}{*}{ EWSR1 rearrangement } & \multicolumn{7}{|c|}{ CD99 positivity } \\
\hline & \multicolumn{3}{|c|}{$<50 \%$} & \multicolumn{3}{|c|}{$\geq 50 \%$} & $P$ value \\
\hline & + & - & IN & + & - & IN & \\
\hline Ewing sarcoma & 2 & 0 & 1 & 17 & 0 & 2 & 0.021 \\
\hline Non Ewing sarcoma & 0 & 6 & 0 & 0 & 10 & 0 & \\
\hline
\end{tabular}

IN: FISH was inconclusive.

certainly the most limiting step and the most difficult. This time setting affects the quality of genetic materials by modifying the structure of nucleic acids (RNA, DNA) as well as proteins [14]. The IHC technique showed low specificity (58\%) and low positive and negative predictive values (48\% and $92 \%$, respectively) compared to the FISH technique (specificity $87 \%$, positive predictive values $89 \%$, and negative $100 \%)$. However, assessment of CD99 immunoreactivity was useful for the selection of EPS cases that may be candidates for molecular testing by FISH. Tumors containing $<50 \%$ of CD99 positive cells had low probability to be rearranged as demonstrated by the $\chi^{2}$ test $(p<0.005)$. CD99 is not specific for Ewing sarcoma and can be positive in other cancers such as poorly differentiated synovial sarcomas, small round cell osteosarcomas, rhabdomyosarcomas, gliomas, and granulosa cell tumors [8]. Therefore, CD99 alone might not be used as an accurate diagnostic immunohistochemical biomarker of EPS. It was suggested that a combination of CD99 and FLI1 may increase the accuracy of IHC [15].

While the FISH break-apart method provided some clear advantages, its main disadvantage is that the translocation partner is not identified, which is important in predicting tumor behavior. As EWSR1 rearrangement may be found in other tumors such as desmoplastic small round cell tumors [16], a detection of fusion types (EWSR1/FLI-1 and EWSR1/ $E R G$ ) from RNA is necessary to confirm the diagnosis using reverse transcription polymerase chain reaction (RT-PCR), which is a highly specific method $[3,17]$. Prognosis of this entity also depends on several factors including the location and volume of the tumor, the stage, the age, and the response to chemotherapy [18]. Current treatment allows 5-year survival of $\sim 70 \%$ for localized stages but less than $35 \%$ in EPS patients with advanced disease [19]. Our study showed that EWSR1 rearrangement and CD99 expression are potential biomarkers for diagnosis of EPS, and more advanced investigation to study their correlations with prognosis, survival, and therapy response are awaited. 


\section{Conclusion}

In order to correctly diagnose EPS, laboratory testing should include both histopathology and CD99 immunostaining, as well as molecular analysis using a dual-color, break-apart rearrangement probe in order to better characterize this entity by identifying the EWSR1 rearrangement. Large prospective series to validate these results are urgently needed especially with the rareness of these highly malignant sarcomas.

\section{Data Availability}

A laboratory notebook explaining all the steps is available in the laboratory. The data is scanned and stored in two external hard drives and available from the corresponding author on request.

\section{Ethical Approval}

All procedures performed in studies involving human participants were in accordance with the ethical standards of the institutional and/or national research committee and with the 1964 Helsinki declaration and its later amendments or comparable ethical standards.

\section{Conflicts of Interest}

The authors declare that they have no conflicts of interest.

\section{Acknowledgments}

The authors thank Professor Sourav Ghosh and Doctor Khalid El Bairi for their contribution to this work. This research was funded by the Hassan II University Hospital and by the Sidi Mohamed ben Abdellah University as part of the preparation of a national thesis $(\mathrm{PhD})$.

\section{References}

[1] J. Ewing, "Diffuse endothelioma of bone," Proceedings of the New York Pathological Society, vol. 21, pp. 17-24, 1921.

[2] C. D. M. Fletcher, J. A. Bridge, P. Hogendoorn, and F. Mertens, Eds., WHO Classification of Tumours of Soft Tissue and Bone, IARC Press, Lyon, 4th edition, 2013.

[3] C. Renard and D. Ranchère-Vince, "Ewing/PNET sarcoma family of tumors: towards a new paradigm?," Annales de Pathologie, vol. 35, no. 1, pp. 86-97, 2015.

[4] E.-Y. K. Choi, J. M. Gardner, D. R. Lucas, J. B. McHugh, and R. M. Patel, "Ewing sarcoma," Seminars in Diagnostic Pathology, vol. 31, no. 1, pp. 39-47, 2014.

[5] J. V. M. G. Bovée and P. C. W. Hogendoorn, "Molecular pathology of sarcomas: concepts and clinical implications," Virchows Archiv, vol. 456, no. 2, pp. 193-199, 2010.

[6] M. Cantile, L. Marra, R. Franco et al., "Molecular detection and targeting of EWSR1 fusion transcripts in soft tissue tumors," Medical Oncology, vol. 30, no. 1, p. 412, 2013.

[7] S. S. Desai and N. A. Jambhekar, "Pathology of Ewing's sarcoma/PNET: current opinion and emerging concepts," Indian Journal of Orthopaedics, vol. 44, no. 4, pp. 363-368, 2010.
[8] M. Tsokos, R. D. Alaggio, L. P. Dehner, and P. S. Dickman, "Ewing sarcoma/peripheral primitive neuroectodermal tumor and related tumors," Pediatric and Developmental Pathology, vol. 15, Supplement 1, pp. 108-126, 2012.

[9] A.-M. Šimundić, "Measures of diagnostic accuracy: basic definitions," EJIFCC, vol. 19, no. 4, pp. 203-211, 2009.

[10] C. Fisher, "The diversity of soft tissue tumours with EWSR1 gene rearrangements: a review," Histopathology, vol. 64, no. 1, pp. 134-150, 2014.

[11] S. K. Kim and Y. K. Park, "Ewing sarcoma: a chronicle of molecular pathogenesis," Human Pathology, vol. 55, pp. 91100, 2016.

[12] B. Llombart, C. Monteagudo, J. A. Lopez-Guerrero et al., "Clinicopathological and immunohistochemical analysis of 20 cases of Merkel cell carcinoma in search of prognostic markers," Histopathology, vol. 46, no. 6, pp. 622-634, 2005.

[13] R. M. Patel, E. Downs-Kelly, S. W. Weiss et al., "Dual-color, break-apart fluorescence in situ hybridization for EWS gene rearrangement distinguishes clear cell sarcoma of soft tissue from malignant melanoma," Modern Pathology, vol. 18, no. 12, pp. 1585-1590, 2005.

[14] P. Mhawech-Fauceglia, F. Herrmann, R. Penetrante et al., "Diagnostic utility of FLI-1 monoclonal antibody and dual-colour, break-apart probe fluorescence in situ (FISH) analysis in Ewing's sarcoma/primitive neuroectodermal tumour (EWS/PNET). A comparative study with CD99 and FLI-1 polyclonal antibodies," Histopathology, vol. 49, no. 6, pp. 569-575, 2006.

[15] Q. Li, W. Cui, G. Abulajiang et al., "Application of immunohistochemistry in the diagnosis of small round blue-cell tumors of soft tissue," Clinical Laboratory, vol. 60, no. 8, pp. 1383-1392, 2014.

[16] A. A. Sandberg and J. A. Bridge, "Updates on cytogenetics and molecular genetics of bone and soft tissue tumors: desmoplastic small round cell tumors," Cancer Genetics and Cytogenetics, vol. 138, no. 1, pp. 1-10, 2002.

[17] J. Noujaim, R. L. Jones, J. Swansbury et al., "The spectrum of EWSR1-rearranged neoplasms at a tertiary sarcoma centre; assessing 772 tumour specimens and the value of current ancillary molecular diagnostic modalities," British Journal of Cancer, vol. 116, no. 5, pp. 669-678, 2017.

[18] The ESMO/European Sarcoma Network Working Group, "Soft tissue and visceral sarcomas: ESMO clinical practice guidelines for diagnosis, treatment and follow-up," Annals of Oncology, vol. 25, Supplement 3, pp. iii102-iii112, 2014.

[19] J. Potratz, U. Dirksen, H. Jürgens, and A. Craft, "Ewing sarcoma: clinical state-of-the-art," Pediatric Hematology and Oncology, vol. 29, no. 1, pp. 1-11, 2012. 


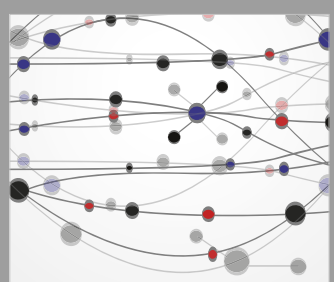

The Scientific World Journal
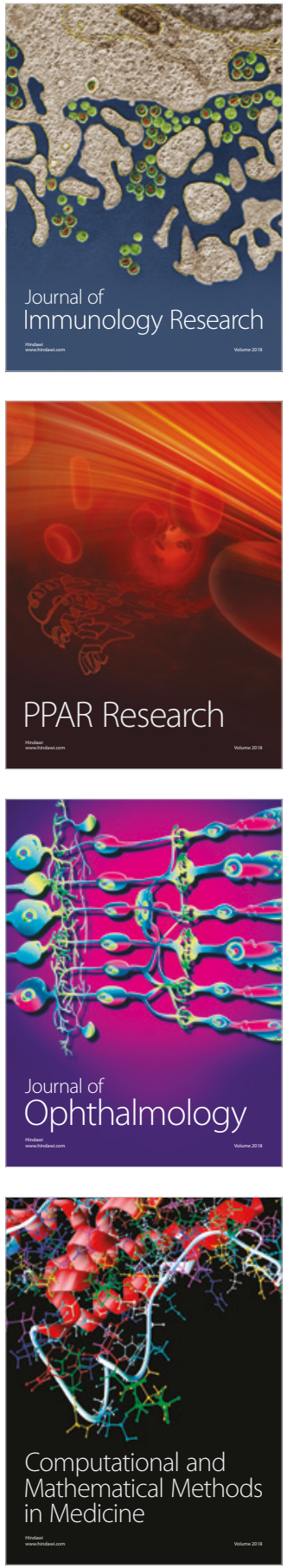

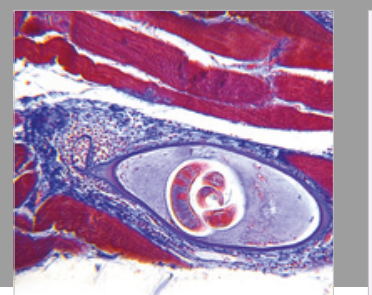

Gastroenterology Research and Practice

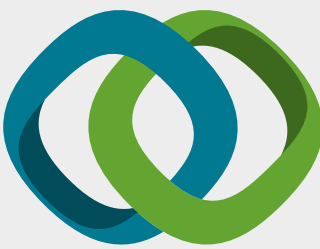

\section{Hindawi}

Submit your manuscripts at

www.hindawi.com
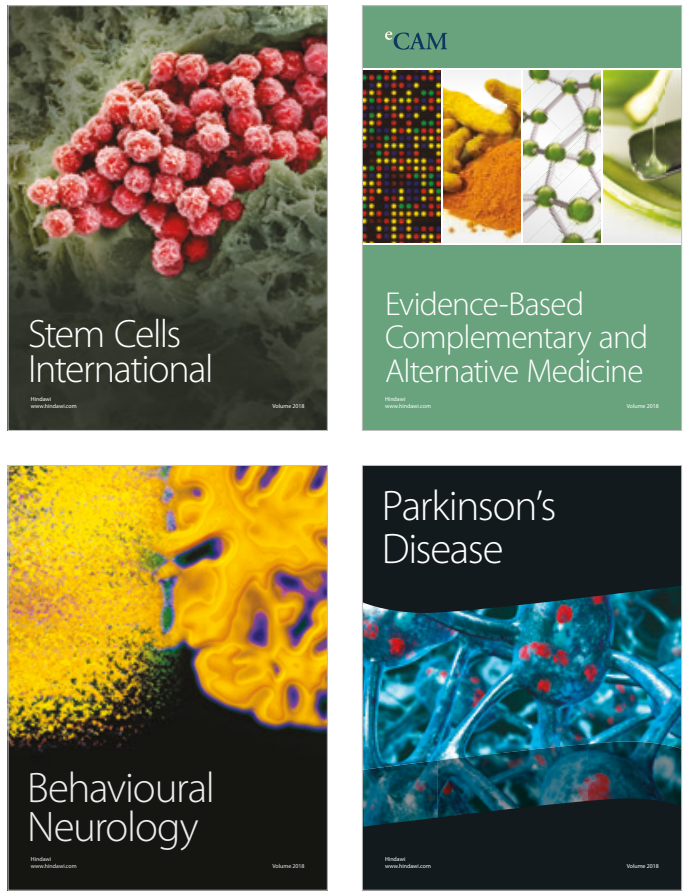

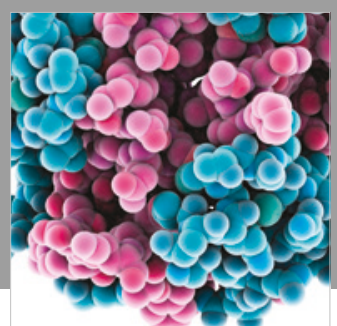

ournal of

Diabetes Research

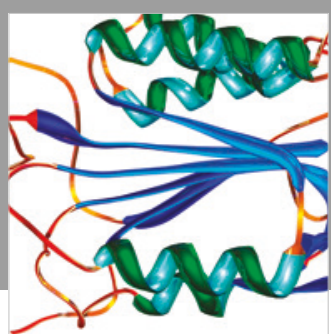

Disease Markers
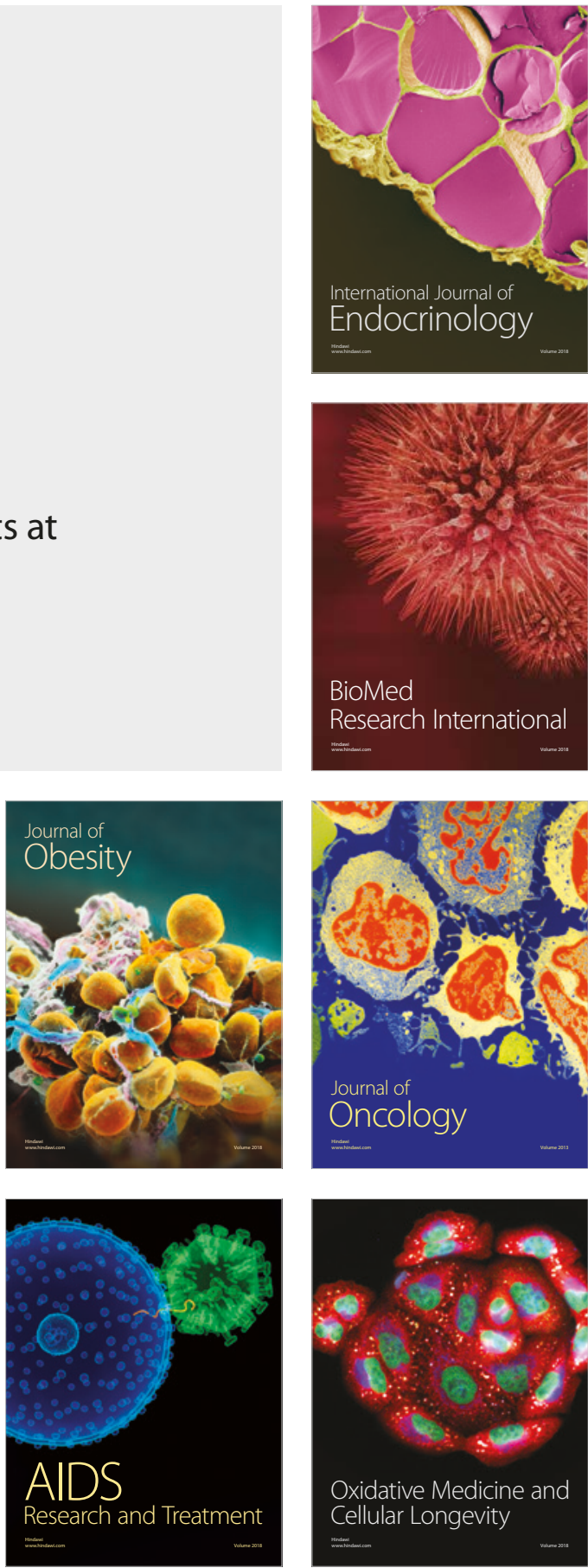